\title{
La movilidad a la salud en las regiones sanitarias VI y VII del conurbano bonaerense
}

\author{
CLARA PIERINI
}

Becaria inicial del Proyecto PICT 2014 - 0693, Instituto del Conurbano, Universidad Nacional de General Sarmiento

clara.pierini@gmail.com

\section{RESUMEN}

El trabajo analiza la movilidad a la salud en las Regiones Sanitarias VI y VII del conurbano bonaerense a partir de los datos de la ENMODO (2009-2010). Los resultados revelan una duración que supera los 3 I minutos en más de la mitad de los viajes y una marcada feminización de la población usuaria. Sobresale la gran cantidad de desplazamientos realizados por personas mayores de 50 años y genera interrogantes el bajo porcentaje de entre o y I 4 años. El cruce de los datos de cobertura de salud y duración de los viajes indica diferencias en el acceso entre los usuarios de los diferentes subsectores. El alto porcentaje de viajes que tuvieron como destino un partido distinto del de residencia denota desigualdades entre los territorios.

Las dificultades que enfrenta la población para movilizarse son, consecuentemente, las que enfrentan mujeres y niños para acceder a la atención. Estas barreras cobran importancia en los territorios elegidos por sus características sociosanitarias, el gran número de mujeres en edad fértil y el alto porcentaje de nacimientos sobre el total del conurbano.

palabras ClaVe: movilidad, salud, Regiones Sanitarias VI y VII, Región Metropolitana de Buenos Aires, Encuesta de Movilidad Domiciliaria 


\section{ABSTRACT}

THE HEALTH MOBILITY IN HEALTH REGIONS VI AND VII OF THE GREATER BUENOS AIRES

This paper analyzes health mobility in Health Regions VI and VII of the Greater Buenos Aires Metropolitan Area, based on the data provided by the ENMODO (2009-2010). The results emphasize a duration that exceeds 31 minutes in more than half of the trips and a marked feminization of those who travel. The large amount of trips made by people over 50 years old stand out while the low percentage of trips made by people from $\circ$ to i 4 year old raise questions. The crossing between of health coverage and travel duration data indicates differences in the access among users of different subsectors. The high percentage of trips that have had a different destination, other than the one of residence, denotes inequalities between the territories.

The difficulties to mobilize faced by the population are, in fact, those faced by women and children to access health. These barriers are relevant in the chosen territories, due to their socio-sanitary characteristics, the large number of women of childbearing age and the high percentage of births (based on the total amount of births of the Greater Buenos Aires Metropolitan Area).

KEYwORDS: mobility, health, Health Regions VI and VII, Greater Buenos Aires Metropolitan Area, Everyday Mobility Survey 


\section{RESUMO}

\section{A MOBILIDADE À SAÚDE NAS REGIÕES SANITÁRIAS VI E VII DA GRANDE BUENOS AIRES}

O trabalho analisa a mobilidade à saúde nas Regiões Sanitárias VI e VII da Grande Buenos Aires, de acordo com dados da ENMODO (2009-2010). Os resultados destacam uma duração que supera os $3 \mathrm{I}$ minutos em mais da metade das viagens e uma destacada feminização da população de usuários. Sobressai o grande número de deslocamentos feitos por pessoas com mais de 50 anos e gera questionamentos a baixa porcentagem entre zero e $\mathrm{I} 4$ anos. O cruzamento de dados de cobertura de saúde e a duração das viagens indica diferenças no acesso entre os usuários dos diferentes subsetores. A alta porcentagem de viagens destinadas a uma festa diferente da de residência denota desigualdades entre os territórios.

As dificuldades enfrentadas pela população para se mobilizar são aquelas enfrentadas por mulheres e crianças para ter acesso a saúde. Essas barreiras são importantes nos territórios escolhidos, devido às suas características socio-sanitárias, o grande número de mulheres em idade fértil e a alta porcentagem de nascimentos no total da Grande Buenos Aires.

PALAVRAS-CHAVE: mobilidade, saúde, Regiões Sanitárias VI y VII, Região Metropolitana de Buenos Aires, Pesquisa de Mobilidade Domiciliária 


\section{Introducción}

Con el propósito de problematizar y caracterizar la movilidad a la salud en las Regiones Sanitarias (RS) VI y VII del conurbano bonaerense,I el trabajo analiza los datos secundarios provistos por la Encuesta de Movilidad Domiciliaria (ENMODO) de la Secretaria de Transporte de la Nación. Esta encuesta fue aplicada en el Región Metropolitana de Buenos Aires (RMBA) entre los años 2009 y 20ıo. Asimismo, el recorrido procura brindar nuevas herramientas para el análisis de dos redes de servicios de salud orientadas a la atención perinatal 2 desplegadas en los territorios seleccionados.

Con estos fines, la propuesta comienza realizando algunas aclaraciones metodológicas y, luego, definiendo los conceptos de «territorio», «transporte», «movilidad» y «movilidad a la salud» y brindando algunos indicadores sociosanitarios de las RS — considerados necesarios para el análisis de la ENMODO-. Luego, se presentan los resultados del procesamiento y algunas reflexiones que surgen de su análisis.

\section{Aclaraciones metodológicas}

Recorte espacio-temporal

La decisión de tomar como unidad de estudio a las Regiones Sanitarias responde a que, en la provincia de Buenos Aires, éstas constituyen espacios descentralizados del Estado provincial en materia salud; son espacios de decisión técnico-política, encargados de la planificación, gestión y/o articulación de políticas sanitarias desde una perspectiva regional. ${ }^{3}$ Privilegiar esta «mirada institucional» permite, además, situar la problemática de la movilidad a la salud dentro de los procesos de regionalización de la atención que vienen desarrollándose en el conurbano bonaerense.

1 La delimitación refiere a los 24 partidos del Gran Buenos Aires que rodean a la Ciudad Autónoma de Buenos Aires.

2 La atención perinatal refiere a la atención materna, fetal y neonatal (OPS-MSAL, 2011).

3 La creación de las Regiones Sanitarias data de 1966 y se modificó en el año 2006 a través del Decreto 3377. En la actualidad, la provincia de Buenos Aires está dividida en doce Regiones Sanitarias. 
Específicamente, la elección de las RS VI y VII refiere a intereses que exceden los objetivos de este trabajo y que se enmarcan en una investigación más amplia sobre las redes de servicios de salud perinatal en estos territorios. Resumidamente, resultan de interés como casos de estudio porque sus territorios poseen las siguientes particularidades: buena presencia de establecimientos de los tres niveles de atención, ${ }^{4}$ la existencia de efectores de las tres jurisdicciones —es una particularidad de los territorios seleccionados, ya que la Nación no posee efectores bajo su órbita en todas las RS-, el funcionamiento de hospitales de alta complejidad ${ }^{5}$ y la implementación del proceso de «regionalización perinatal». ${ }^{6}$ Por otra parte, ambas RS presentan alta densidad poblacional y un alto porcentaje de nacimientos sobre el total provincial y del conurbano. ${ }^{7}$ Estos atributos hacen a las RS comparables entre sí, aunque presentan particularidades en términos de indicadores relativos a la población, la salud perinatal y la estructura sanitaria - Tablas i y 2.

4 Los sistemas de salud presentan tres niveles de atención. El primero presta servicios orientados a la prevención, diagnóstico, tratamiento y rehabilitación, en especialidades básicas y de modalidad ambulatoria. El segundo brinda prestaciones y servicios a usuarios que requieren atención ambulatoria especializada o internación. Finalmente, el tercero, se caracteriza por brindar prestaciones de alta complejidad (Bonazzola, 2009).

5 Referimos, en la RS VI, el Hospital de Alta Complejidad «El Cruce»y, en la RS VII, al Hospital Nacional Posadas.

6 Desde 2010 se ha trabajado a nivel nacional en la iniciativa de «regionalización perinatal», que apunta a mejorar el acceso a la salud y la cobertura, y supone el desarrollo de un sistema de salud materno-infantil descentralizado, con niveles de cuidados y con efectores trabajando en red. Esta propuesta procura que las mujeres y niños obtengan la atención que necesitan, según el riesgo (OPS-MSAL, 2011).

7 En el año 2010, la RS VI tenía el mayor porcentaje de nacimientos sobre total provincial, que corresponde al $26 \%$, y casi el $43 \%$ de los partos del conurbano; a la RS VII correspondían, para el mismo año, aproximadamente el $15 \%$ de los nacimientos provinciales y el $22 \%$ de los del conurbano - este porcentaje excluye los datos de los partidos de la RS que no pertenecen al conurbano- . Las cifras corresponden al subsector oficial y son una elaboración propia, realizada a partir de los datos de la Dirección de Información Sistematizada del Ministerio de Salud provincial. 
Tabla 1. Indicadores relativos a la población y a la salud perinatal. RS VI y VII, provincia de Buenos Aires. Año 2010. ${ }^{8}$

\begin{tabular}{|c|c|c|c|c|}
\hline & RS VI & & $\begin{array}{l}\text { RS VII } \\
\text { (solo partidos del } \\
\text { conurbano) }{ }^{1}\end{array}$ & \\
\hline & Valores absolutos & $\%$ & Valores absolutos & $\%$ \\
\hline Población* & 3768994 & - & 1991.244 & - \\
\hline Total de hogares* & 1122346 & - & 597788 & - \\
\hline Total de hogares con NBI* & 108059 & 9,63 & 47936 & 8,02 \\
\hline $\begin{array}{l}\text { Población con cobertura } \\
\text { de obra social, plan } \\
\text { privado y/o mutual* }^{*}\end{array}$ & 2270760 & 60,25 & 1205482 & 60,54 \\
\hline Nacidos vivos** & 74703 & - & 36732 & - \\
\hline $\begin{array}{l}\text { Tasa de mortalidad } \\
\text { infantil }{ }^{\star \star}\end{array}$ & 12,4 & - & 12,9 & \\
\hline Defunciones maternas ** & 29 & - & 29 & - \\
\hline $\begin{array}{l}\text { Tasa de mortalidad } \\
\text { materna** }^{*}\end{array}$ & 3,88 & - & 7,88 & - \\
\hline
\end{tabular}

Fuente: *Censo 2010, INDEC. **Dirección de Información Sistematizada del Ministerio de Salud de la Provincia de Buenos Aires, 2010.

Tabla 2. Indicadores relativos a la estructura sanitaria del subsector público. RS VI y VII, provincia de Buenos Aires, 2010.

\begin{tabular}{|c|c|c|c|c|c|}
\hline & & \multicolumn{2}{|l|}{ RS VI } & \multicolumn{2}{|c|}{$\begin{array}{l}\text { RS VII } \\
\text { (solo partidos del } \\
\text { conurbano) }\end{array}$} \\
\hline & & $\begin{array}{l}\text { Valores } \\
\text { absolutos }\end{array}$ & $\%$ & $\begin{array}{l}\text { Valores } \\
\text { absolutos }\end{array}$ & $\%$ \\
\hline \multirow{2}{*}{ Efectores } & Efectores con internación & 27 & - & 12 & - \\
\hline & Efectores sin internación & 358 & - & 131 & - \\
\hline \multirow{4}{*}{ Camas } & Camas & 3893 & - & 1767 & - \\
\hline & Camas municipales & 404 & 10,4 & 558 & 31,6 \\
\hline & Camas provinciales & 3487 & 89,6 & 740 & 41,9 \\
\hline & Camas nacionales & 3 & 0,1 & 469 & 26,5 \\
\hline
\end{tabular}

Fuente: Dirección de Información Sistematizada del Ministerio de Salud de la Provincia de Buenos Aires, 2010.

8 En la sistematización de indicadores sociodemográficos, de salud y de estructura sanitaria se han considerado datos del 2010, por ser el año en que se terminó la recolección de datos de la ENMODO. 
La RS VI es una de las más habitadas, con una población de 3747 486 habitantes (Censo, 20Io), lo que representa el 37,8 \% de la población del conurbano. Como da cuenta el Mapa I, incluye a los municipios de Almirante Brown, Avellaneda, Berazategui, Esteban Echeverría, Ezeiza, Florencio Varela, Lanús, Lomas de Zamora y Quilmes. En el año 20Io, el subsector público de salud estaba compuesto por I6 hospitales provinciales, 4 hospitales municipales y una Unidad de Pronta Atención (UPA) de dependencia provincial.

La RS VII está integrada por los municipios de General Las Heras, General Rodríguez, Luján, Marcos Paz, Merlo, Hurlingham, Ituzaingó, Morón, Tres de Febrero y Moreno (Mapa I). Los municipios de General Las Heras, General Rodríguez, Luján y Marcos Paz no forman parte del conurbano bonaerense. La Región tiene una población estimada de 2253772 habitantes. Vale mencionar que los seis partidos que forman parte del conurbano, en su conjunto, alcanzan los I 99I 244 habitantes, es decir, 19,4I \% de la población de los 24 partidos del Gran Buenos Aires (Censo, 20ıo). En el año 20ı, el subsector público de salud de estos 6 partidos la RS VII estaba compuesto por un hospital nacional, 5 hospitales provinciales y 6 hospitales municipales.

Fuente: elaboración propia con datos provenientes de la guía de establecimientos 2010 de la Dirección de Información Sistematizada del Ministerio de Salud de la Provincia de Buenos Aires.

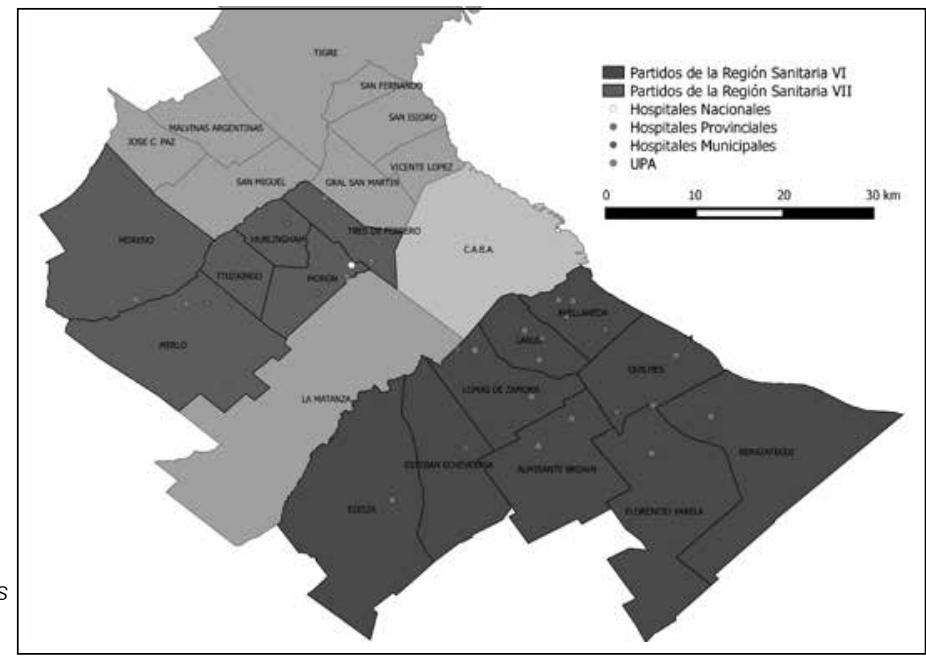

Mapa 1. Localización de los hospitales (nacionales, provinciales y municipales) y UPAS en las Regiones Sanitarias VI y VII del conurbano bonaerense. Año 2010. 


\section{La Encuesta de Movilidad Domiciliaria}

Los datos analizados provienen de la ENMODO, que fue aplicada en el Región Metropolitana de Buenos Aires (RMBA) entre los años 2009 y 20Io. Se trató de una iniciativa de la Secretaría de Transporte de la Nación, que procuró caracterizar de modo cuantitativo algunos aspectos vinculados con la movilidad de la población de la mencionada área. Específicamente, la encuesta recogió datos de los viajes y sus etapas en los territorios relevados, como así también algunas características de las personas y hogares encuestados. Si bien esta información permite problematizar y analizar la movilidad cotidiana — como daremos cuenta a lo largo del desarrollo de la propuesta-, está centrada en el transporte y atiende particularmente a los trayectos de origen y destino.

En la RMBA se encuestó a una muestra representativa de 22170 hogares - encuestas efectivas - y 70 321 personas (Ministerio de Transporte de la Nación, s/f). Se indagó acerca de las características socioeconómicas de cada hogar y sus integrantes y, por supuesto, sobre la movilidad. Se encuestó a la totalidad de los habitantes de cada hogar que hubieran realizado viajes el día anterior al que se realizó la ENMODO. Específicamente, en la RS VI se encuestaron 567I hogares y I3 201 personas, y en la VII 303I hogares y 707I personas.

El trabajo de campo de la ENMODO fue acotado a la Ciudad Autónoma de Buenos Aires (CABA) y 27 partidos de la provincia de Buenos Aires - Vicente López, San Isidro, General San Martín, Tres de Febrero, Hurlingham, Ituzaingó, Morón, La Matanza, Lomas de Zamora, Lanús, Avellaneda, Quilmes, Almirante Brown, Esteban Echeverría, Ezeiza, Merlo, Moreno, San Miguel, José C. Paz, Malvinas Argentinas, San Fernando, Tigre, Escobar, Pilar, Presidente Perón, Florencio Varela y Berazategui-, pero el aérea de influencia del estudio se extiende a los 42 de la RMBA. La selección fue definida en relación a la continuidad de la trama urbana y la cantidad de población - los partidos elegidos concentran del $90 \%$ de la población de la RMBA- (Ministerio de Transporte de la Nación, s/f). Vale destacar que algunos de los partidos correspondientes a la RS VII no formaron parte de la muestra: General Las Heras, General Rodríguez, Luján y Marcos Paz, por lo que no serán analizados en el presente estudio.

La base completa de la ENMODO se encuentra disponible en la página web de la Unidad Ejecutora Central del Ministerio de Trans- 
porte de la Nación (Ministerio de Transporte de la Nación, s/f). Los datos se encuentran desagregados en cuatro partes: Hogares, personas, viajes y etapas. Para el presente artículo se trabajó con las bases de personas, viajes y etapas. En primer lugar, el registro de viajes se recortó en función de los motivos de viaje, dado el objetivo de analizar específicamente la movilidad a la salud. Luego, los viajes a la salud fueron unidos a las características de las personas que los realizaron a través de la variable «ID Persona» presente en ambas bases. La base «Etapas» fue acotada a aquellas correspondientes a viajes por motivos de salud, utilizando la variable «ID Viajes». El procesamiento fue realizado con la ayuda de los softwares para análisis de datos cuantitativos Excel y SPSS.

El desarrollo de este trabajo considera las siguientes variables en relación a los viajes y sus etapas: cantidad de etapas, frecuencia, horario de salida, duración, medio/s de transporte utilizados, actividad de origen, partido de destino, etc. Respecto de las características de los usuarios encuestados por la ENMODO indaga sobre su sexo, edad y cobertura de salud. En el análisis, los datos para las Regiones Sanitarias VI y VII — siempre que es posible - son contrastados con los de la RMBA, provistos por el informe que realizó el Ministerio de Transporte (s/f) a partir de la ENMODO.

Finalmente, merecen atención algunas limitaciones de la ENMODO. $\mathrm{Si}$ bien no se desconoce que sus resultados son muy valiosos dados los escasos aportes empíricos que abordan — cuantitativamente y con amplio alcance - el transporte y la movilidad en la RMBA y, de manera puntual, la movilidad a la salud, existe una serie de dificultades para trabajar con sus resultados. En primer lugar, la desagregación de los resultados en cuatro bases hace difícil, por su diseño, volver a unirlas para trabajar los datos en conjunto y, así, se dificulta la obtención rápida y sencilla de información sobre movilidad. En segundo lugar, el documento teórico-metodológico de la encuesta es acotado, omite el instrumento utilizado para el relevamiento y prescinde de información acerca de algunos criterios utilizados en la selección de los datos. ${ }^{9}$

9 Por ejemplo, se desconoce si la encuesta indaga sobre satisfacción de necesidades o la llegada a lugares, aunque todo pareciera indicar que se trabaja sobre la segunda opción. 


\section{Aportes conceptuales para abordar la movilidad}

La definición territorio: hacia una mirada sistémica, relacional y relativizada

El análisis que se presenta a continuación requiere trascender las miradas fragmentadas que perciben el territorio solo como un espacio geográfico o de competencias, distinguido por su homogeneidad, estabilidad y rigidez. De acuerdo con esta tradición, las características del territorio son objetivas y posibles de ser captadas desde un lugar externo, ya que dependen del ambiente físico y las características histórico-culturales instaladas.

Por el contrario, el territorio debe ser entendido como un espacio socialmente producido donde diversos flujos de relaciones articulan y tensionan un conjunto de productos y servicios en un espacio particular - en el cual las distancias físicas se ven relativizadas- - Se trata de un ámbito complejo, virtual y dinámico, maleable a los intercambios, a las relaciones de poder y a la acción de los actores sociales. Esta perspectiva requiere de una visión sistémica que considere distintas escalas y niveles del territorio (Dematteis, 2002; Brugué et al., 2002; Silveira, 2003; Dematteis y Governa, 2005; Blanco, 2009).

Estrechamente vinculada a la mencionada propuesta, existe una línea de trabajo que considera el paso del espacio al territorio como resultado de la acción de un actor específico. Cuando un actor se apropia de un espacio - de modo concreto o abstracto-, lo territorializa y, así, el territorio se concibe como un espacio donde se proyecta un trabajo. Esta línea permite ahondar sobre el papel que desempeña el territorio en el proceso de construcción de una identidad colectiva de los agentes (Silveira, 2003; Dematteis y Governa, 2005). Sus aportes permiten reflexionar sobre la definición de territorio asociada al análisis de redes de políticas y, específicamente, de redes de servicios de salud.

Gran parte de las propuestas asociadas a la mirada relativizada y relacional del territorio utiliza el paradigma de las redes como una metáfora para el análisis (Blanco, 2009; Lévy, 2009). Blanco (2009:4) afirma que no existe territorio sin red, ya que las redes permiten la movilidad, el establecimiento de lazos y la comunicación: «se hace necesario pensar las redes en relación con el conjunto de objetos técnicos ya fijados en el territorio, y con el conjunto de prácticas y estrategias que despliegan los actores sobre el territorio». Retoma la idea de «territorios-red», que permite pensar la multiterritorialidad —entendida como la conexión entre territorios diversos y la posibilidad de pasaje de un territorio a 
otro- y, consecuentemente, integrar lógicas reticulares y areales. Lévy (2009) agrega que este par permite dar cuenta de las distintas métricas en los nuevos territorios urbanos y reflejar las proximidades invisibles. ${ }^{10}$

En cuanto a los elementos que conforman las redes, las propuestas de los geógrafos Blanco (2009) y Silveira (2003) — quienes retoman al brasilero Milton Santos - se alejan de las miradas que solo atienden a los aspectos materiales de las redes y proponen que también debe considerarse el dato social. La red «é também social e política, pelas pessoas, mensagens, valores que a freqüentam» (Santos, 1996:209). Así, Blanco (2009) indica que las redes tienen una arquitectura formal y una organización social, y asigna a las redes tres componentes: infraestructura - elementos materiales_- flujos — dan cuerpo a las redes mediante la utilización de la infraestructura- e infoestructura — marco regulatorio que permite su funcionamiento.

Esta mirada sobre el territorio se vuelve necesaria para pensar la movilidad ya que, como se verá en el próximo apartado, brinda herramientas para complejizar los desplazamientos más allá de sus características materiales y objetivas.

Articulaciones y tensiones entre la movilidad y el transporte

Los nuevos paradigmas en materia de movilidad se centran en las personas más que en los medios de transporte. En esta línea, Lévy (2009) y Gutiérrez (20I2) rechazan la idea de considerar la movilidad como un mero desplazamiento que puede conocerse a partir de la configuración material del territorio. Gutiérrez (2012, 2013) sostiene que la reflexión sobre la movilidad requiere su distinción del transporte, aunque ambos conceptos comparten su objeto de estudio: el desplazamiento de las personas y sus bienes en un territorio, tiempo y contexto social específicos. El transporte es un concepto restringido, vinculado a una concepción material del espacio y refiere al vector o medio a través del cual se

10 También han reflexionado sobre la propuesta de «territorios-red» quienes indagan sobre las nuevas formas de gobierno y territorialidad producto de la movilidad creciente del capital y la información, que se traduce en novedosas relaciones económicas, sociales y políticas. Estas producciones destacan el papel de los territorios locales y de los actores en los procesos de desarrollo (Brugué et al., 2002; Dematteis, 2002; Pié, 2002; Dematteis y Governa, 2005). 
realiza un desplazamiento. La movilidad supone un entendimiento más amplio del espacio y describe una práctica social de desplazamiento de personas tanto físicas como jurídicas.

Lévy (2009) explica que, si bien la movilidad contempla también una dimensión material —el viaje realizado-, como hecho social implica considerar dimensiones virtuales e ideales: la accesibilidad, las posibilidades de movilidad efectiva y el capital social. Con accesibilidad el autor se refiere a la oferta material de movilidad - u oferta de transporte- - ya que la posibilidad de una persona de acceder a un lugar es clave para pensar su movilidad, sin que esto signifique que irá a todos los lugares accesibles. Menciona la existencia de una relación triangular entre tres polos que ejercen influencia unos sobre otros: lo deseado, lo posible y lo realizado efectivamente. La movilidad efectiva no refiere solamente a la posesión de los medios económicos, sino también a una "competencia de movilidad» que implica, por ejemplo, tener en cuenta la red de lugares frecuentados por los diversos grupos considerados. Lévy explica que la movilidad, en ocasiones, debe ser estimulada para ofrecer a ciertos sectores oportunidades —incluso las gratuitas-. El capital social está relacionado con las dimensiones ya mencionadas. Con relación a sus posibilidades de movilidad de movilidad efectiva y a la accesibilidad, cada individuo inventa su ciudad — privilegiando ciertos lugares y eliminando otros.

A partir de esta noción de movilidad, Gutiérrez (2011, 2012, 2013) distingue entre los enfoques ontológicos y los teleológicos, que suponen dos definiciones de viaje — unidad de estudio- - El primero supone que el viaje es lineal, ya que une dos puntos, y que se sucede en un tiempo continuo, aunque su tiempo de duración es variable. El enfoque teleológico, centrado en las personas, considera que el fin de la movilidad no es llegar a un lugar sino la satisfacción de un deseo o necesidad. Así, la morfología del viaje es reticular — puede incluir más de dos lugares- y discontinua — plausible de realizarse en más de un día.

Vale mencionar que la movilidad cotidiana — sobre la que trabaja la ENMODO - está asociada a los viajes que realizan las personas diariamente por motivos laborales, de educación, de salud, de recreación, compras, entre otros. Consecuentemente, se torna imprescindible para la reproducción social. Esta movilidad, si bien es territorial, condiciona la movilidad social —ascenso o descenso de una posición social, económica o cultural—, ya que permite la inclusión o exclusión de las personas a la 
sociedad: «las oportunidades de la vida en sociedad están mediadas por las condiciones de la movilidad territorial» (Gutiérrez, 20II:I55).

Finalmente, interesa distinguir entre los términos acceso y accesibilidad. La accesibilidad es un atributo de los lugares y refiere a la facilidad - $\mathrm{O}$ no- con que se llega a un lugar. Por el contrario, el acceso es una noción centrada en las personas. Entiende que los individuos, incluso cuando viven en un mismo lugar y comparten características socioeconómicas, pueden tener acceso diferencial a distintos lugares, actividades, bienes y servicios. El acceso incluye al transporte, pero también depende aspectos personales y familiares, de la actividad o servicio que se busca satisfacer, del contexto sociohistórico, entre otras cuestiones. El acceso - como la movilidad - no responde a una distancia material, acceder refiere a la satisfacción del fin que buscaba el viaje (Gutiérrez:I56).

La definición de movilidad denota una concepción del territorio sistémica, que supone distintas escalas y niveles, y la integración de lógicas áreales y reticulares. Esta mirada sobre el territorio permite dar cuenta de métricas diversas, como también de cercanías y distancias invisibles, que es preciso tener en cuenta a la hora de pensar en movilidad. Esta implica una consideración del territorio que excede el plano de lo material y objetivo para pensarlo como una construcción constante, en íntimo juego con los procesos y actores implicados.

\section{La movilidad a la salud}

Siguiendo la propuesta anteriormente planteada, este trabajo entiende la movilidad a la salud como aquellos desplazamientos que buscan satisfacer necesidades de salud. Están orientados a alcanzar actividades de prevención, diagnóstico, tratamiento, rehabilitación y cuidados paliativos desarrolladas en el ámbito de los servicios de salud ${ }^{11}$ (Chiara, 20I3).

Como señala Gutiérrez (20II, 20I3), para el caso de la salud materna en zonas periurbanas de la RMBA, el acceso a la salud describe viajes en red —no lineales-. Estos viajes encadenados están orientados a un mismo servicio. Identificar esta red es importante, ya que da cuenta del acceso

11 Las actividades orientadas a la promoción de la salud fueron excluidas, ya que por su intención de abordar los determinantes sociales de la salud transcienden el ámbito de los servicios de salud (Chiara, 2013). 
a la salud por parte de una población específica. Muestra que el acceso no está signado simplemente por la proximidad física y el transporte costo, tiempo de viaje, etc.- sino también por las características de los servicios y efectores, la calidad de la atención, aspectos personales, entre otras cuestiones. Por estas razones, la unidad de estudio de la movilidad a la salud también es el viaje, entendido como el nexo material entre una necesidad o deseo y la realización de la actividad o servicio que la satisface (Gutiérrez, 20II, 20I2, 20I3). Es preciso mencionar que la perspectiva teórica propuesta abre dimensiones difíciles de trabajar con los datos provistos por la ENMODO. Estas dimensiones, mayormente vinculadas a lo simbólico, requieren de abordajes cualitativos.

El transporte y la movilidad aparecen como un problema en los diagnósticos de políticas sanitarias, aunque las estrategias propuestas en esta área son escasas. La idea de movilidad que predomina en estos informes es aquella que la entiende como un mero desplazamiento que puede conocerse a partir de la configuración material del territorio (Gutiérrez, 2013).

Así, Gutiérrez (20II, 20I3) destaca la necesidad de trabajar con una noción de movilidad centrada en las personas en vistas a garantizar un enfoque de derechos en las políticas públicas en general y en las de transporte y salud en particular, ya que condiciona la inserción de las personas en la vida social. La movilidad es relevante para la agenda pública de transporte y también para la sanitaria, ya que el acceso a los servicios de salud se encuentra condicionado por la misma, lo que da cuenta de la necesidad de intervenciones intersectoriales. Este énfasis cobra particular relevancia para la Argentina, donde el derecho a la salud es una garantía constitucional.

Para el caso de las RS seleccionadas, resulta de interés complejizar algunos datos indicados en el apartado metodológico en vistas a reflexionar sobre la movilidad a la salud. Ambas regiones presentan altos porcentajes de hogares con NBI, mostrando la RS VI un porcentaje aún mayor que la RS VII -9,63 y 8,02 \%, respectivamente-. En este plano las diferencias entre partidos son amplias, los datos del Censo 2010 muestran jurisdicciones con porcentajes menores a los de su RS, como Lanús en la VI (5 $\%$ y Morón en la VII (3,5\%), y otros que lo superan ampliamente, como Florencio Varela en el caso de la VI (I7 \%) y Moreno en la VII (I2,9 \%). Además, en ambas regiones aproximadamente un $40 \%$ de la población sólo cuenta con cobertura de salud del subsector estatal. Aquí las diferencias entre partidos también son notorias, hay partidos con un porcentaje 
cercano al $50 \%$, como Florencio Varela y Moreno, y otros con alrededor del 30 \%, como Morón, Ituzaingó, Lanús y Avellaneda. Estos datos dan cuenta de un alto porcentaje de la población en situación de vulnerabilidad social -mayormente en los partidos de la segunda corona del conurbano- ${ }^{12} \mathrm{y}$, en consecuencia, con grandes dificultades para desplazarse - por el gasto que supone, deficiencias en la urbanización de sus barrios de residencia, falta de acceso a información, etc.

La oferta de servicios también determina las posibilidades de movilidad a la salud de la población. Particularmente, la oferta hospitalaria de la provincia de Buenos Aires posee un conjunto heterogéneo de efectores cuya infraestructura y composición tecnológica varía según dependencia, ${ }^{13}$ especialidad y función en la red de servicios. En la provincia de Buenos Aires también es necesario el análisis sobre la dotación de camas, ya que el $79 \%$ de los hospitales públicos con internación depende de los gobiernos municipales pero, por su menor tamaño y complejidad, solo se encuentran bajo su jurisdicción el $50 \%$ de las camas (San Martín y Luzuriaga, 2012).

Como muestra el Mapa I, la oferta hospitalaria del subsector público varía entre las RS analizadas, como así también al interior de las mismas - hay partidos que cuentan con tres o cuatro hospitales en su jurisdicción y algunos que poseen solo un efector municipal e, incluso, ninguno-. Un estudio de San Martín y Luzuriaga (20I2) que elabora un índice de complejidad, que no solo considera la estructura hospitalaria sino también la capacidad instalada, permite sostener que los hospitales de mayor complejidad se encuentran en los partidos aledańos a la Ciudad Autónoma de Buenos Aires - Lomas de Zamora, Avellaneda y Morón—, con excepción del Hospital de Alta Complejidad «El

12 Se distinguen tres coronas en el Gran Buenos Aires, en relación a su proximidad respecto de la Ciudad Autónoma de Buenos Aires. La primera y la segunda corona corresponden a los partidos que conforman el conurbano bonaerense. La primera corona está compuesta por los partidos de Avellaneda, Quilmes, Lanús, Lomas de Zamora, La Matanza I, Tres de Febrero, Morón, Ituzaingó, Hurlingham, General San Martín, Vicente López y San Isidro. La segunda corona refiere a los partidos de Berazategui, Florencio Varela, Almirante Brown, Esteban Echeverría, Ezeiza, La Matanza II, Merlo, Moreno, San Miguel, José C. Paz, Malvinas Argentinas, Tigre y San Fernando.

13 Los hospitales de dependencia provincial tienen una infraestructura que les permite realizar prácticas más complejas, asociadas a equipos y tecnología que se justifican en una mayor escala de producción hospitalaria que la que brindan los efectores municipales (San Martín y Luzuriaga, 2012). 
Cruce» de Florencio Varela. Esta situación, sumada a los indicadores sociosanitarios de la segunda corona conurbano, denota posibilidades de acceso y movilidad desiguales al interior de las RS. Por otra parte, la Tabla 2 muestra un importante peso de los municipios en la RS VII, donde el 3I,6 \% de las camas se encuentra bajo su dependencia. En ciertas ocasiones, la dependencia municipal del segundo nivel de atención atenta contra la calidad de la atención, ya que dificulta la resolución de prestaciones de mayor complejidad con independencia de las autoridades locales, el acceso a recursos críticos y la construcción de redes de servicios — que favorecen el tránsito de los usuarios por los distintos niveles del sistema- (Chiara et al., 20II).

\section{La movilidad a la salud en dos RS del conurbano bonaerense}

La presente sección analiza la movilidad a la salud en los territorios seleccionados. En línea con este objetivo se procura dar respuesta a los siguientes interrogantes: ¿cómo son los procesos de movilidad a la salud en las RS VI y VII del conurbano bonaerense? ¿Qué cantidad de etapas conllevan los viajes por motivos de salud? ¿Cuánto duran? ¿Cómo se distribuyen según el horario? ¿Cuál es su frecuencia? ¿Aparecen variaciones según el sexo, la edad y la cobertura de salud de la población? ¿Qué porcentaje de viajes salen del partido de residencia en busca de atención? ¿Cuáles son los medios de transporte utilizados más asiduamente? Vale aclarar que las respuestas a estas preguntas, a continuación, atienden a las diferencias entre las RS.

Antes de avanzar sobre el mencionado objetivo, resulta interesante atender al peso de los viajes a la salud sobre el total de viajes, que en ambas RS se encuentra por debajo del promedio para la RMBA. Específicamente, los viajes por motivos de salud representan 2,4\% del total de viajes de la RS VI ( $n=30$ 194) y $2,5 \%$ de la RS VII ( $n=16$ I42). En la RMBA representan el 4,2\% del total de viajes (Ministerio de Transporte de la Nación, s/f). De aquí en adelante los datos que se analicen refieren a los viajes por motivos de salud.

Recuperando los interrogantes de otros estudios que analizan encuestas de movilidad cotidiana (Ministerio de Transporte de la Nación, s/f; Puebla y Palomares, 2006), nos preguntamos en primer lugar la cantidad de etapas que suponen los viajes por motivos de salud. Las 
etapas refieren a un cambio en el modo de transporte o vehículo dentro de un mismo viaje. Por ejemplo, quien para llegar al trabajo primero toma un tren y luego un colectivo, hace un viaje en dos etapas.

Las regiones bajo estudio presentan algunas diferencias en este punto, presentando la RS VII una mayor cantidad de viajes realizados en más de una etapa. En la RS VI el 83,I \% de los viajes se resuelven en una sola etapa, el I2,4 \% en dos etapas y el 4,5\% en tres. ${ }^{14}$ En la RS VII, los viajes de una sola etapa representan el 78,3\%, los de dos etapas el I7,5 \% y los de tres el 4,2\%. Es notable que los datos RS VI no muestren grandes variaciones respecto del total de viajes en la RMBA, donde un $85 \%$ se resuelven en una sola etapa, un $13 \%$ se realiza en dos etapas y un $2 \%$ en tres etapas (Ministerio de Transporte de la Nación, s/f). La RS VII muestra algunas diferencias: los viajes que se realizan en una sola etapa representan un porcentaje menor, aumentando los que se realizan en dos y tres etapas.

En cuanto a las diferencias de género, Jirón (2007:I74) explica que "cómo hombres y mujeres experimentan de manera diferenciada la movilidad, tiene consecuencias en la vida cotidiana y la calidad de vida urbana». En línea con esta propuesta, consideramos de interés analizar los viajes en relación al sexo. En las RS VI y VII los viajes por motivos de salud muestran una marcada feminización, ya que más del 60\% corresponde a mujeres — ver Gráfico I-. Es posible vincular esta situación a la mayor presencia y utilización de los servicios de salud por parte de las mujeres - situación que no se traduce en una expansión de la perspectiva de género- (MSAL, 20II; Pozzio, 20II). Esta tendencia no se manifiesta a nivel de la RMBA, donde no aparecen diferencias entre varones y mujeres (Ministerio de Transporte de la Nación, s/f).

14 El n varía según la unidad de análisis: cuando se analizan los viajes por motivos de salud en la RS VI el n es 738 y en la RS VII corresponde a 405; cuando se caracteriza a las personas que viajaron, en la RS VI el n es 718 y en la RS VII corresponde a 390. Esta situación aparece porque hay personas que realizaron más de un viaje en un día. 


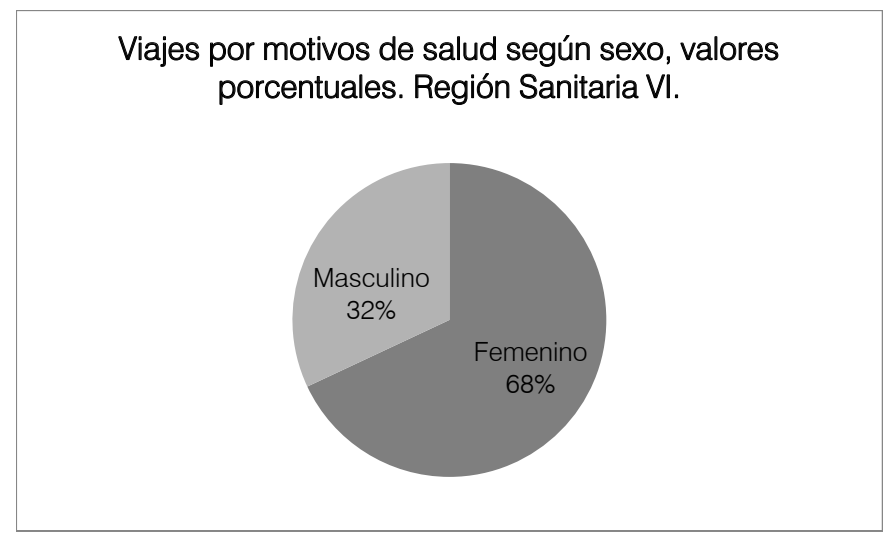

Viajes por motivos de salud según sexo, valores porcentuales. Región Sanitaria VII.

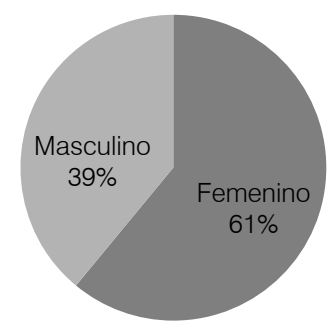

Fuente: elaboración propia sobre la base de datos de la ENMODO 20092010, Secretaría de Transporte de la Nación.

Gráfico 1. Viajes por motivos de salud según sexo, valores porcentuales. RS VI y VII, provincia de Buenos Aires, 2009-2010.

La ENMODO también permite conocer la cantidad de viajes que realizó una persona el día anterior a realizar la encuesta. La frecuencia de los viajes por motivos de salud en el caso de las RS analizadas es baja, ya que aproximadamente el $97 \%$ de los entrevistados ${ }^{15}$ indicó haber realizado sólo un viaje. En este sentido, es preciso aclarar que su

15 A lo largo del texto se utilizarán sustantivos en masculino (usuario, entrevistado) para no entorpecer la lectura con barras (usuario/a, entrevistado/a). Vale destacar que en todos los casos se refiere a personas de distintos géneros. 
baja frecuencia no es necesariamente negativa, sino que se asocia a las características de la movilidad a la salud. Así, no es posible comparar su frecuencia con la de otras movilidades - por ejemplo, la movilidad laboral supone, normalmente, frecuencias mucho más altas- . Por otra parte, el informe del Ministerio de Transporte de la Nación (s/f) para la RMBA señala que la frecuencia con que se realizan viajes por motivos de salud es baja: el $69 \%$ tiene un carácter eventual (su frecuencia es menor a una vez a la semana) y el i2 \% una frecuencia semanal.

Fuente: elaboración propia sobre la base de datos de la ENMODO 20092010, Secretaría de Transporte de la Nación.
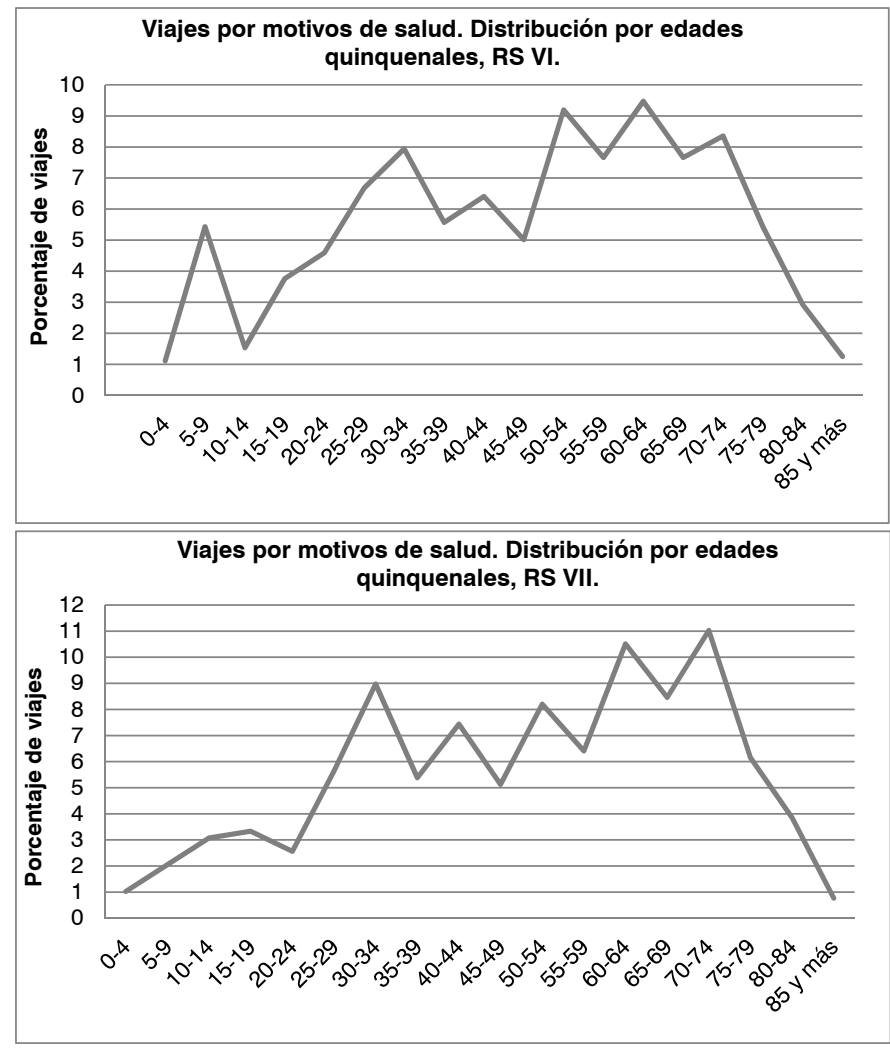

Gráfico 2. Viajes por motivos de salud. Distribución según edades quinquenales. RS VI y VII, provincia de Buenos Aires, 2009-2010. 
Otro de los aspectos tomados en cuenta fue la distribución de los viajes por edades quinquenales, particularmente por el interés de indagar en las redes perinatales en ambas $\mathrm{RS}$ - cuyos usuarios son, principalmente, mujeres en edad fértil y neonatos-. En primer lugar, observando el Gráfico 2, llama la atención el bajo porcentaje sobre el total que significan los viajes entre los o y I4 años, a pesar de la gran cantidad de controles obligatorios para esta franja etaria. Los viajes entre los I5 y 49 años, rango en el que se encuentran las mujeres en edad fértil, corresponden aproximadamente al $40 \%$ en ambas regiones. Luego, es notable el aumento en la cantidad de viajes entre los 50 y 74 años en la RS VI y entre los 60 y 74 en la VII. Vale destacar que en la RS VI el $5 \mathrm{I}, 9 \%$ de los viajes los realizaron personas mayores de 50 años y en la RS VII el 55,4\%.

En cuanto a la cobertura más del $60 \%$ de los encuestados mencionó poseer una obra social en ambos territorios bajo análisis. Luego, le sigue la población cubierta por el sector público y aquellos afiliados a la medicina prepaga. Específicamente, en la RS VI, el 60,7 \% de las personas entrevistadas tenían cobertura de obras sociales, el 5,8\% a través de medicina prepaga y el I, $3 \%$ por mutuales. El 31,6 \% de la muestra no poseía cobertura de salud, por lo que utilizaba los servicios que brinda el sector público. En la RS VII el 69,2 \% de la muestra poseía cobertura a través de alguna obra social, 4,9\% mediante medicina prepaga y el o,3\% a través de mutuales. Respecto de los usuarios de los servicios públicos, fueron el $25,4 \%$ de la muestra.

Interesa considerar que en ambas RS el porcentaje de población con cobertura, calculado a partir de las encuestas realizadas, es más alto que los valores arrojados por el Censo 2010 - ver Tabla I-. También es posible comparar estos datos con los de la Encuesta Nacional de Protección y Seguridad Social (ENAPROSS), realizada en el año 2oıı por el Ministerio de Trabajo, Empleo y Seguridad Social. De acuerdo con la ENAPROSS, en el conurbano, el $47,4 \%$ de los habitantes recibe cobertura a través obras sociales, II,7 \% mediante al PAMI/PROFE y 2,6 \% por seguros privados. La cobertura brindada por efectores públicos representaba el 38,2\%.

Con relación al lugar de inicio de los desplazamientos, en ambas regiones se menciona el hogar en $94 \%$ de los casos. En segundo lugar, con un valor cercano al $2 \%$, aparece como origen el lugar de trabajo o la resolución de algún asunto laboral. Llama la atención que la salud aparece como origen del desplazamiento en $1,5 \%$ de los viajes para ambas regiones. Pero si analizamos el caso de las personas que han reali- 
zado más de un viaje por salud este porcentaje aumenta —si bien no es estadísticamente significativo, ya que el porcentaje de personas que realizaron dos o más viajes es muy bajo- : en la RS VI ocupa el primer lugar con un $50 \%$ y en la RS VII el segundo con un $40 \%$.

El Gráfico 3 permite conocer la distribución del horario de salida de los viajes por motivos de salud en las áreas bajo estudio. Es posible distinguir que los desplazamientos comienzan a aumentar luego de las 6 de la mañana, produciéndose el pico más alto a las 8 en la RS VI y a las 9 en la RS VII. Luego de otro pico entre las I5 y I6 h, según la RS, el descenso de los viajes es marcado y durante la noche prácticamente no se comienzan viajes. Es posible asociar esta distribución con el horario de funcionamiento de la mayor parte de los servicios de salud - excluyendo aquellos que atienden urgencias-, entre las 8 y las $16 / 17 \mathrm{~h}$. En el caso de la RMBA, los picos se presentan alrededor de las io de la mañana y le siguen las ir y las I2. Luego, llaman la atención los picos de las 9, I5 y i6 h (Ministerio de Transporte de la Nación, s/f).

Fuente: elaboración propia sobre la base de datos de la ENMODO 20092010, Secretaría de Transporte de la Nación.
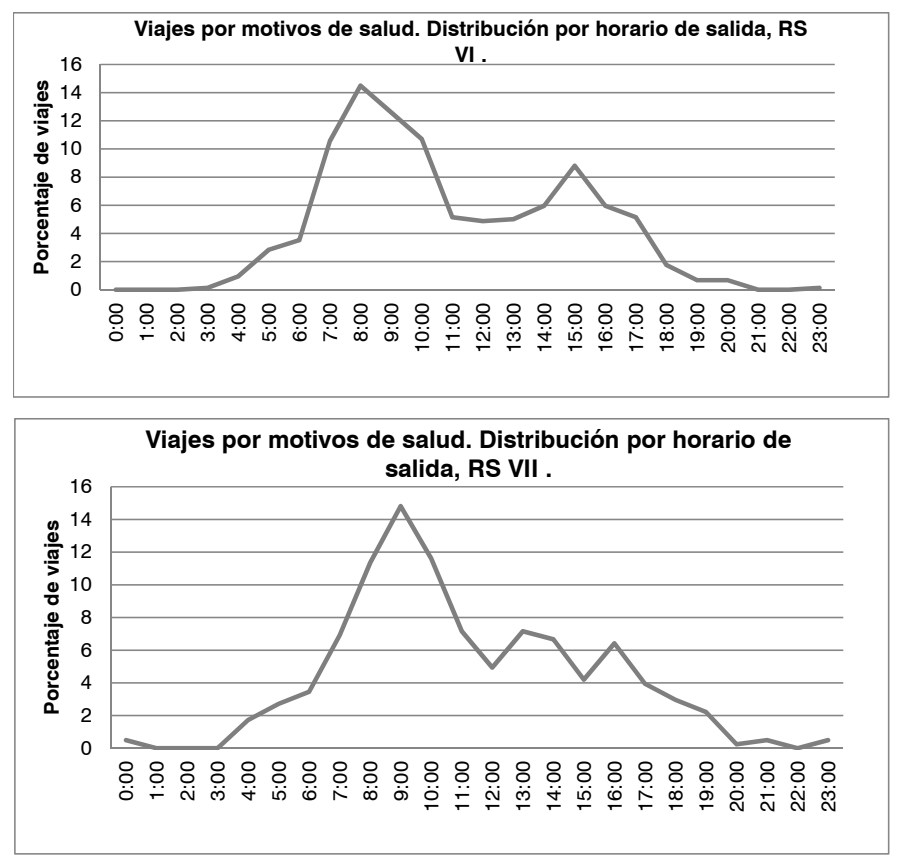

Gráfico 3. Viajes por motivos de salud. Distribución por horario de salida. RS VI y VII, provincia de Buenos Aires, 2009-2010 
La duración de los viajes por motivos de salud fue otro eje de análisis. En este punto, las diferencias entre ambas RS no son marcadas e interesa destacar que en ambas aproximadamente la mitad de los viajes tienen una duración de más de 3I minutos y cerca del $20 \%$ supone más de una hora - Gráfico 4-. En la RS VI, el $32 \%$ de los viajes por motivos de salud tiene una duración de entre 3I minutos y una hora; el $22 \%$, entre 2I y 30 minutos; el $20 \%$, entre II y 20 minutos; el I7 \%, más de una hora de viaje, y el $9 \%$ toma hasta Io minutos. En la RS VII, el 3I \% dura entre 3I minutos y una hora, el $22 \%$ entre 2I y 30 minutos, el 2I \% más de una hora, el i $6 \%$ entre ir y 20 minutos y el Io $\%$ hasta Io minutos.

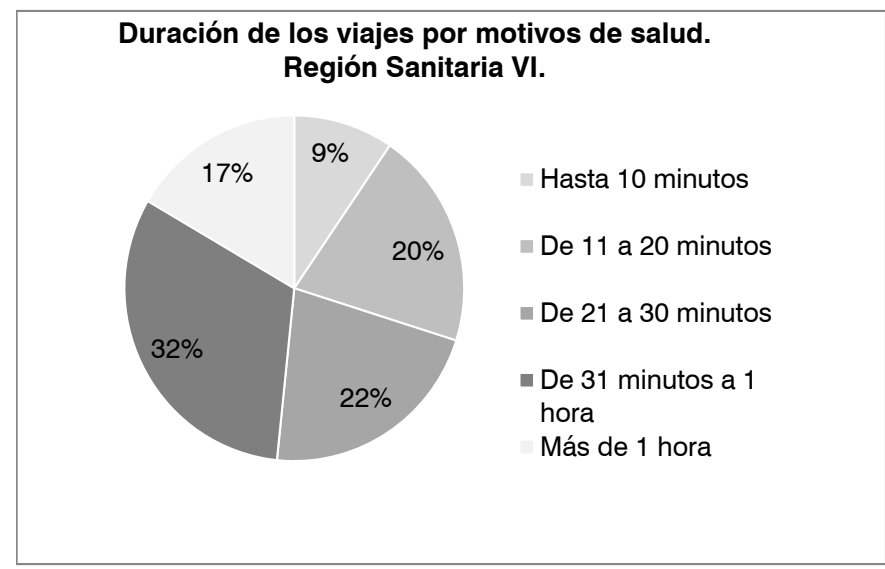

\section{Duración de los viajes por motivos de salud. Región Sanitaria VII.}

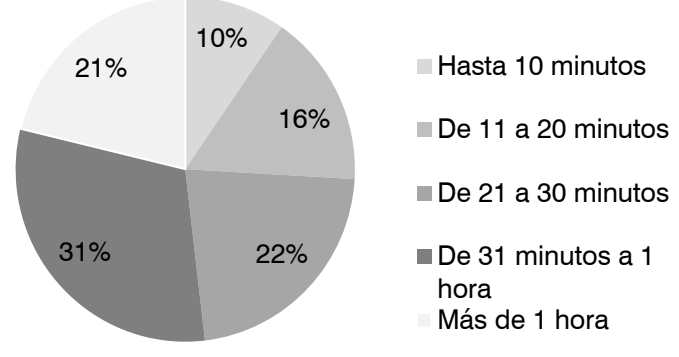

Fuente: elaboración propia sobre la base de datos de la ENMODO 20092010, Secretaría de Transporte de la Nación.

Gráfico 4. Duración de los viajes por motivos de salud. RS VI y VII, provincia de Buenos Aires, 2009-2010. 
Como da cuenta el Gráfico 5, la duración de los viajes en la RMBA no presenta grandes diferencias con los resultados obtenidos para las RS analizadas. Allí, la duración de los viajes por motivos de salud presenta variaciones según quintil de ingreso, siendo que la representación de los quintiles más bajos en los viajes más largos es mayor que la de los quintiles altos. Esta relación se invierte en los viajes más cortos (Secretaria de Transporte de la Nación, s/f:98-99). Dado a que en el presente trabajo se han analizado directamente las encuestas de la ENMODO no es posible cruzar los datos sobre la duración de los viajes por quintil de ingresos pero, a modo de aproximación al nivel de ingresos, se han utilizado los datos de cobertura de salud (Gráfico 6).

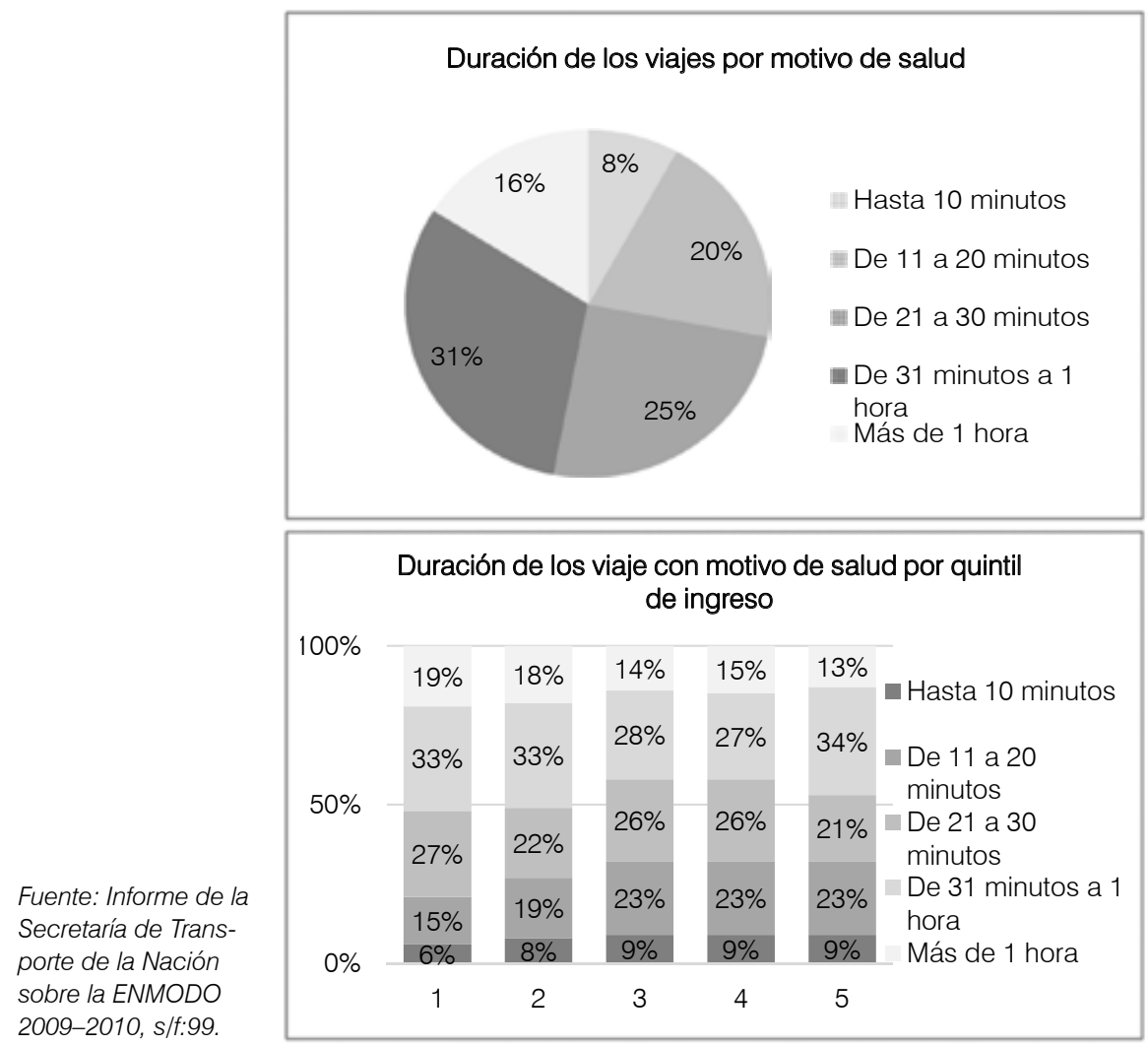

Gráfico 5. Duración de los viajes por motivos de salud y su variación según quintil de ingreso. Región Metropolitana de Buenos Aires. 

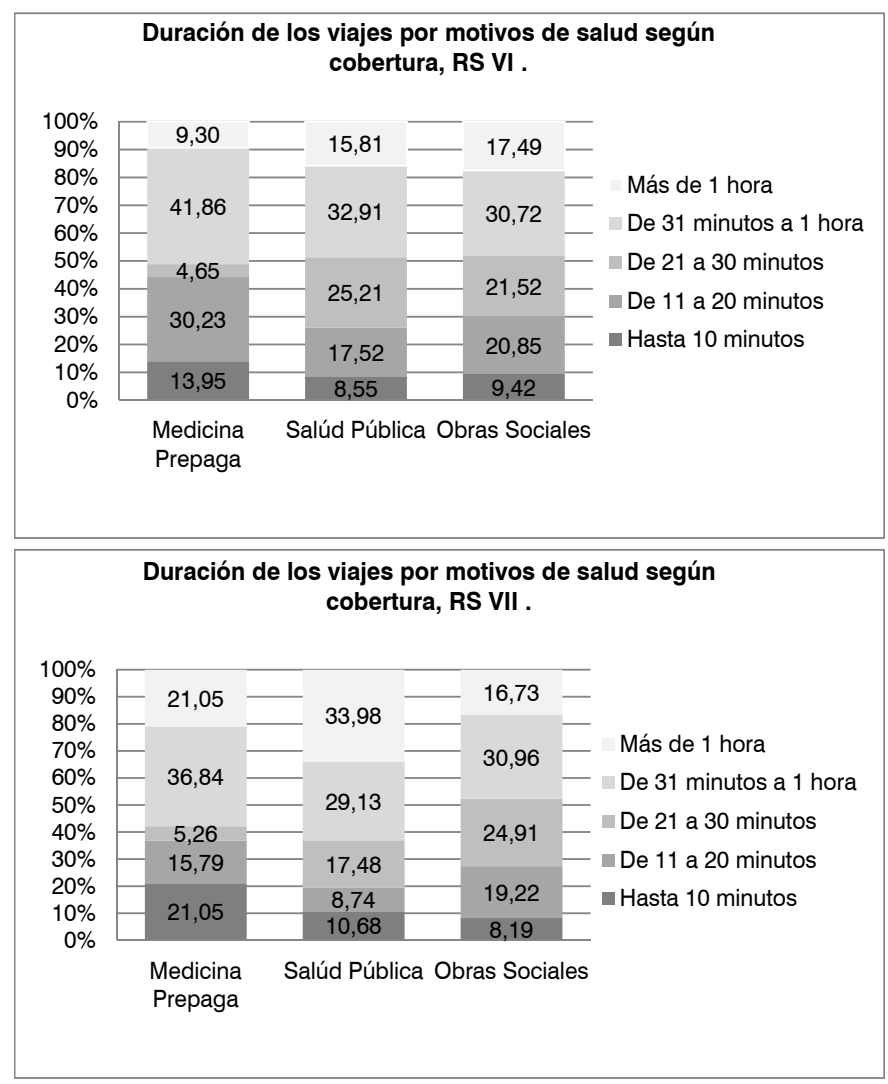

Fuente: elaboración propia sobre la base de datos de la ENMODO 20092010, Secretaría de Transporte de la Nación.

Gráfico 6. Duración de los viajes por motivos de salud según cobertura. RS VI y VII, provincia de Buenos Aires, 2009-2010.

De acuerdo con el Gráfico 6, son notables las diferencias entre subsectores. Particularmente, el $34 \%$ de los viajes a la salud pública en la RS VII dura más de una hora, siendo que los viajes de esta duración para aquellas personas cubiertas por obras sociales descienden al $16,7 \% \mathrm{y}$, en el caso de los usuarios de la medicina prepaga, al 2I,I \%. En esta área, el 63,I \% de los viajes de los usuarios del sector público tarda más de $3 \mathrm{I}$ minutos en llegar a los servicios de salud, mientras que este valor asciende al 48,7 \% en las RS VI. En la RS VI, la duración de los viajes entre los usuarios de los servicios públicos y de las obras sociales no presentan variaciones importantes, aunque sí respecto de aquellos que asisten a servicios de medicina prepaga. 
La duración de los viajes, asimismo, fue analizada con relación al partido de destino del viaje. Los datos de la ENMODO destacan que en la RS VI el $44,9 \%$ de los viajes por motivos de salud ha tenido como destino un partido distinto al de residencia y, en la RS VII, un 5I,I \%. Como permite visualizar el Gráfico 7, el cruce de estas variables da cuenta que los viajes fuera del partido de residencia son más largos, representando en ambas RS alrededor del $90 \%$ de los viajes de más de una hora y cerca del $60 \%$ de aquellos entre 31 y 60 minutos. Estos resultados dan cuenta de la fragmentación y la segmentación del sistema sanitario en el conurbano, como así también de la desigualdad de los territorios —en términos de estructura sanitaria, equipamientos, transporte, urbanización, entre otros- (Chiara et al., 20II), que redundan en diferentes barreras de acceso a la salud. En este contexto, la población en muchas oportunidades se ve obligada a salir de su partido de residencia en busca de atención.

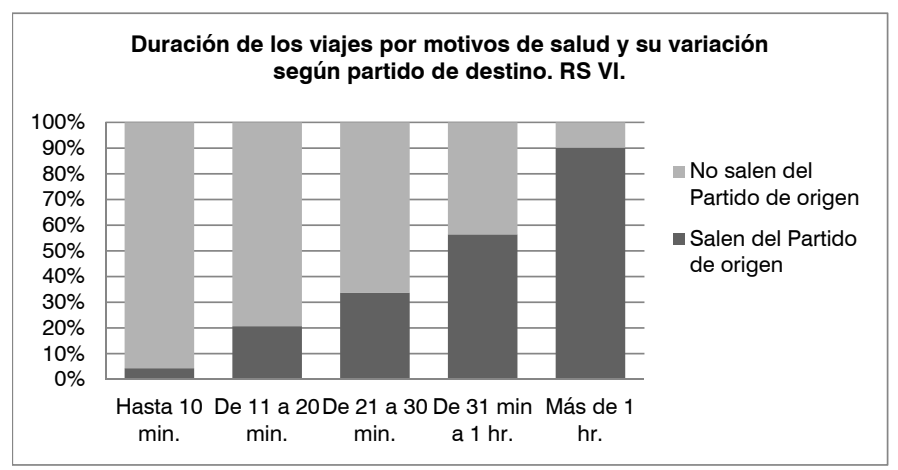

Fuente: elaboración propia sobre la base de datos de la ENMODO 20092010, Secretaría de Transporte de la Nación.

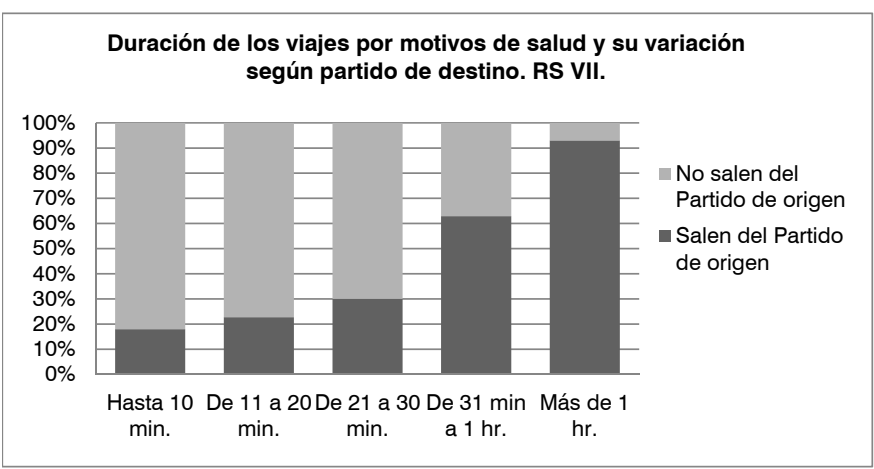

Gráfico 7. Duración de los viajes por motivos de salud y su variación según partido de destino. RS VI y VII, provincia de Buenos Aires, 2009-2010. 
A partir de los datos del Grafico 7, surge el interés de considerar el porcentaje de viajes que salen del partido de residencia en busca de atención tomando como unidad de análisis las jurisdicciones que forman parte de las RS VI y VII. Los datos que muestra la Tabla 3 dan cuenta de diversas situaciones según el partido que se considere - que en ciertos casos pueden vincularse con la dotación de estructura sanitaria- y de realidades disímiles al interior de ambas RS. En la RS VI, Quilmes y Ezeiza presentan los porcentajes más bajos de viajes fuera del partido - $22 \%$ y $33,3 \%$, respectivamente-, que en el caso de Quilmes plantean una serie de interrogantes dado la presencia de un solo hospital y su lejanía respecto de la CABA y los partidos del primer cordón del conurbano, que poseen mayor estructura de servicios. Los partidos que en esta Región poseen más de un 50\% de los viajes fuera de su jurisdicción despiertan distintas reflexiones según su ubicación y estructura sanitaria. Lanús, Lomas de Zamora y Avellaneda presentan un amplio porcentaje de viajes fuera del partido, a pesar de contar con elevado número de efectores en comparación con otras jurisdicciones del conurbano. La no elección de los mismos abre múltiples interrogantes sobre la calidad de la atención, la disponibilidad de los servicios, su estrategia de Atención Primaria de la Salud, entre otros, y, también, puede asociarse a su proximidad a la CABA. Es posible vincular el caso de Florencio Varela a la escasa dotación de estructura sanitaria del partido. En la RS VII, el alto porcentaje de viajes fuera del partido en Ituzaingó puede explicarse por la ausencia de hospitales en la jurisdicción y en el caso de Hurlingham por la presencia de un solo efector municipal. Por otra parte, el partido de Morón muestra el porcentaje más bajo de viajes fuera del partido, que puede asociarse a la presencia de tres hospitales en el distrito. ${ }^{16}$

16 Es preciso mencionar que las reflexiones no han considerado la dotación de efectores del primer nivel de atención según partido, por la dificultad para trabajar con estos datos en un trabajo acotado. 
Tabla 3. Viajes según partido de destino. Valores Porcentuales. RS VI y VII, provincia de Buenos Aires.

\begin{tabular}{l|l|l|l|l|l}
\hline $\begin{array}{l}\text { Partidos de la } \\
\text { RS VI }\end{array}$ & $\begin{array}{l}\text { Viajes fuera } \\
\text { del partido } \\
\text { de residencia }\end{array}$ & $\begin{array}{l}\text { Viajes dentro } \\
\text { del partido } \\
\text { de residencia }\end{array}$ & $\begin{array}{l}\text { Partidos de la } \\
\text { RS VII }\end{array}$ & $\begin{array}{l}\text { Viajes } \\
\text { fuera del } \\
\text { partido de } \\
\text { residencia }\end{array}$ & $\begin{array}{l}\text { Viajes dentro } \\
\text { del partido } \\
\text { de residencia }\end{array}$ \\
\hline Brown & 45,5 & 54,5 & Hurlingham & 68,4 & 31,6 \\
\hline Avellaneda & 50,6 & 49,4 & Ituzaingó & 68,8 & 31,3 \\
\hline Berazategui & 45,6 & 54,4 & Merlo & 39,2 & 60,8 \\
\hline E. Echeverría & 45,9 & 54,1 & Moreno & 52,2 & 47,8 \\
\hline Ezeiza & 33,3 & 66,7 & Morón & 39,4 & 60,6 \\
\hline Fcio. Varela & 51,7 & 48,3 & 3 de Febrero & 52,3 & 47,7 \\
\hline Lanús & 52,5 & 47,5 & Total & 51,1 & 48,9 \\
\hline L. de Zamora & 54,2 & 45,8 & & & \\
\hline Quilmes & 22,0 & 78,0 & & & \\
\hline Total & 44,9 & 55,1 & & & \\
\hline
\end{tabular}

Fuente: elaboración propia sobre la base de datos de la ENMODO 2009-2010, Secretaría de Transporte de la Nación.

Por último, en el análisis de los medios de transporte utilizados en los viajes por motivos de salud, no se destacan grandes diferencias entre las RS. Como presenta la Tabla 4, en ambos territorios se destaca el colectivo en primer lugar, con alrededor del $60 \%$, lo que marca una amplia distancia con el resto de las opciones. El segundo lugar lo ocupa el auto, seguido del ferrocarril, el taxi o remís y, finalmente, el transporte a pie. Vale recordar que un viaje puede incluir más de un medio de transporte y, consecuentemente, cada desplazamiento puede tener más de una etapa.

Tabla 4. Medios de transporte utilizados en los viajes por motivos de salud. Valores Porcentuales. RS VI y VII, provincia de Buenos Aires.

\begin{tabular}{l|l|l}
\hline \multicolumn{1}{l}{ RS VI } & \multicolumn{1}{l}{ RSVII } \\
\hline Colectivo & $59,1 \%$ & $58,6 \%$ \\
\hline Auto & $11,7 \%$ & $12,0 \%$ \\
\hline Ferrocarril & $9,4 \%$ & $12,2 \%$ \\
\hline Remís/Taxi & $9,3 \%$ & $7,3 \%$ \\
\hline A pie & $7,5 \%$ & $6,5 \%$ \\
\hline Otros & $3,0 \%$ & $3,5 \%$ \\
\hline
\end{tabular}

Fuente: elaboración propia sobre la base de datos de la ENMODO 2009-2010, Secretaría de Transporte de la Nación 


\section{Conclusiones}

Una mirada del territorio sistémica — capaz de dar cuenta de distintas escalas y de la integración de lógicas areales y reticulares, como así también de métricas diversas y de cercanías y distancias invisiblesse vuelve necesaria para pensar la movilidad; en especial en espacios tan fragmentados y desiguales como los del conurbano bonaerense, ya que brinda herramientas para complejizar los desplazamientos más allá de sus características materiales y objetivas. Esta definición de territorio permite pensar en una movilidad centrada en las personas que, como práctica social de desplazamiento, supone un entendimiento más amplio del espacio; su fin último no es llegar a un lugar sino la satisfacción de un deseo o necesidad. Así, la morfología del viaje es reticular y discontinua - por esta razón, por ejemplo, no solo se atiende a los viajes sino también a sus etapas- (Gutiérrez, 20II, 2012, 2013).

Sobre el tema específico de este trabajo, Gutiérrez (20II,20I3) explica que el acceso a la atención de la salud describe viajes en red y entiende que este acceso no está signado simplemente por la proximidad física y el transporte sino también por las características de los servicios, la calidad de la atención y aspectos personales. En esta línea, se ha considerado a la movilidad a la salud como aquellos desplazamientos que buscan satisfacer necesidades de salud; $y$ se ha tomado como unidad de estudio al «viaje», comprendido como el nexo material entre una necesidad o deseo y la realización de la actividad o servicio que la satisface (Gutiérrez, 20I2).

Particularmente, los territorios de las RS VI y VII presentan características disímiles y son desiguales a su interior. Los indicadores sociosanitarios considerados permiten esperar diversas movilidades y los datos de estructura sanitaria dan cuenta de diferentes posibilidades de acceso a los servicios por parte de los usuarios y/o familias. Así, por ejemplo, la movilidad a la salud en los partidos que cuentan con escasa oferta de servicios y donde más de la mitad de la población está cubierta únicamente por el sector público no se asemejará a la de aquellos con mejores indicadores sociosanitarios, mayor disponibilidad de servicios y una ubicación próxima a la CABA. Los datos de la ENMODO deben analizarse considerando estas características —en especial en los partidos de la segunda corona, como Moreno y Florencio Varela, donde las brechas en el acceso se profundizan. ${ }^{17}$

17 Para un análisis pormenorizado ver Chiara et al., 2011. 
En cuanto al análisis de datos de la ENMODO, en primer lugar, interesa destacar que los viajes por motivos de salud representan en ambas RS alrededor del $2,5 \%$ sobre el total de viajes. Con referencia a la frecuencia de estos viajes, el análisis denota que es baja, ya que aproximadamente el $97 \%$ de los entrevistados indicó haber realizado solo un viaje en la semana bajo estudio. Los horarios de los desplazamientos están vinculados al horario de funcionamiento de la mayor parte de los servicios de salud, entre las 8 y las $17 \mathrm{~h}$. En ambas RS, alrededor del $60 \%$ de los desplazamientos es en colectivo. También, vale la pena señalar que más de la mitad de los viajes tienen una duración de más de 3I minutos. Con relación a la actividad de origen de los viajes, se destaca en ambas regiones el hogar como lugar de inicio del desplazamiento aproximadamente en el $94 \%$ de los casos.

Por otra parte, los viajes en torno a la atención de la salud muestran una marcada feminización en los territorios seleccionados. Esta situación da cuenta de una mayor utilización de los servicios sanitarios por parte de la población femenina, lo que coincide con los resultados otras investigaciones en la RMBA (Di Virgilio, 2003; López y Findling, 2012; Ariovich y Jiménez, 20I4). Aunque excede a este artículo la reflexión acerca del porqué, si es posible alertar que a la hora de pensar la movilidad a la salud deben considerarse las particularidades y vulnerabilidades de la población que utiliza los servicios. Por otra parte, la marcada feminización de los viajes podría explicar el alto porcentaje de desplazamientos que tienen como lugar de partida el hogar.

También debe atenderse a la edad de los usuarios de los servicios. En ambas RS más de la mitad de los viajes fueron realizados por personas mayores de 50 ańos. En tanto, genera interrogantes el bajo porcentaje sobre el total que significan los viajes entre los o y I4 años, a pesar de la gran cantidad de controles obligatorios en esta franja etaria.

Respecto de la cobertura, en la RS VI el 3I,6 \% de la muestra no poseía cobertura de salud, por lo que utilizaba los servicios que brinda el sector público. En la RS VII fueron aproximadamente el 25,4 \% de la muestra. El porcentaje restante gozaba de algún tipo de cobertura —obra social, mutual o prepaga- El cruce de estos datos con la duración de los viajes permite señalar diferencias en el acceso entre los usuarios de los diferentes subsectores, particularmente en la RS VII — donde más del 60 $\%$ de los usuarios del sector público demora al menos 3I minutos para acceder a los servicios de salud. 
Asimismo, la duración de los viajes fue analizada según el partido de destino. Los resultados de la ENMODO destacan que en la RS VI el $44,9 \%$ de los viajes por motivos de salud han tenido como destino un partido distinto al de residencia y, en la RS VII, un 5I,I \%. Los viajes fuera del partido de residencia son más largos, representando en ambas RS alrededor del $90 \%$ de los viajes de más de una hora y cerca del 60 $\%$ de aquellos entre 3ry 60 minutos. Desde una mirada local, la consideración del porcentaje de viajes que salen del partido tomando como unidad de análisis el partido de residencia del usuario, muestra importantes oscilaciones según la jurisdicción. Así, los resultados dan cuenta de la fragmentación y la segmentación que caracterizan al sistema sanitario del conurbano, como también de la desigualdad de los territorios en términos de oferta prestacional, ${ }^{18}$ que redundan en diferentes barreras de acceso a la atención en salud. Esta situación, al vincularse con la dispersión de los hospitales en el conurbano y, más precisamente, con la mayor disponibilidad de servicios en los partidos del primer cordón y la CABA, remite a la noción de «territorio-red» — por la necesidad de considerar no solo lógicas de proximidad, sino también reticulares-. $\mathrm{Y}$ abre numerosos interrogantes acerca de dimensiones vinculadas a lo simbólico, que resultaría interesante indagar a través de técnicas como las biografías de viajes.

Esta última reflexión también origina algunos interrogantes en cuanto a las necesidades que procuran resolver los viajes por motivos de salud analizados. ¿Qué demandan los usuarios que viajan? ¿Hacia qué tipo de efector se dirigen? Estas distinciones serían claves para pensar el porcentaje de los viajes que sale de su partido de residencia en busca de atención. La estrategia de Atención Primaria de la Salud (APS) ${ }^{19}$ promueve el fortalecimiento del primer nivel de atención y, considerando este enfoque, la Organización Panamericana de la Salud sostiene que la mayor parte de necesidades y las demandas de atención deberían resolverse en este nivel (OMS/OPS, 2007). Así, la mayor parte de las

18 Ver: Chiara et al., 2011; Chiara, 2012.

19 El enfoque de APS promueve la organización de sistemas de salud que busquen la equidad y la solidaridad, y, así, que respeten el derecho a la salud en su nivel más alto. Esta meta requiere el desarrollo de integrados e integrales que den respuesta a las necesidades de toda la población sin distinciones, considerando la capacidad del sector y el contexto nacional (OMS/OPS, 2007). 
consultas tendrían realizarse en los partidos de residencia de los usuarios, pero si sus desplazamientos fueran en busca de atención especializada o internación resulta posible que su satisfacción se encuentre en otro partido. ${ }^{20}$

Finalmente, es preciso señalar que las dificultades que enfrenta la población para movilizarse son, consecuentemente, las que enfrentan las mujeres y niños para acceder a la atención. Estas barreras cobran central importancia en ambas RS, no solo por las características sociosanitarias de su población, sino también por el gran porcentaje de mujeres en edad fértil —más del $25 \%$ de las personas que habitan en ambas regiones son mujeres entre is y 49 años-, la alta proporción de nacimientos - sobre el total provincial y del conurbano- y el importante número de partos en establecimientos del subsector público — aproximadamente 35 de cada 1000 mujeres en edad fértil han tenido partos en efectores públicos durante el año 20I0-. ${ }^{21}$ En consecuencia, interesa destacar algunos resultados del estudio que tienen particulares implicancias a la hora de pensar la atención perinatal y que serán profundizados en el trabajo de campo de carácter cualitativo. La larga duración de los viajes -más de la mitad exceden los 3i minutos- es una barrera en el acceso para las mujeres, entre otras razones, porque tienen niños a cargo, que muchas veces viajan con ellas, y porque suelen ocuparse de las tareas del hogar. Esta situación se ve agravada para las usuarias del sector público, particularmente en la RS VII, lo que no puede desestimarse dada su situación de mayor vulnerabilidad. Por último, el alto porcentaje de desplazamientos fuera del partido de residencia, abre numerosos interrogantes dado que, en el caso de las embarazadas, los controles deberían realizarse en el primer nivel de atención — siempre que se trate de un embarazo de bajo riesgo-. En este sentido, la propuesta de redes de servicios de salud ${ }^{22}$ aparece como una estrategia interesante para trabajar

20 Es preciso recordar que la atención en las RS se encuentra - 0 , al menos, esa es su intención- regionalizada y, así, los efectores de mayor complejidad tienen una población de referencia que excede al partido en el que se localizan.

21 Los datos provienen del Censo 2010 y de los informes de ese mismo año de la Dirección de Información Sistematizada del Ministerio de Salud de Provincia de Buenos Aires.

22 Aquellos abordajes preocupados por la aplicación del «modelo de redes» al campo de la planificación y gestión en salud, plantean que las redes involucran una articulación de organizaciones — públicas, privadas, sociedad civil- que brindan un continuo coordinado e integral de servicios de salud para un población y territorio específicos. Estas redes 
las barreras en el acceso a la salud, ya que su fortalecimiento permitiría —entre otras cuestiones — «agilizar» los recorridos de los usuarios dentro del sistema sanitario.

\section{Agradecimiento}

Agradezco a Ana Ariovich por la lectura pormenorizada del artículo y sus aportes.

están también a cargo de los costos y resultados —clínicos, económicos y del estado de salud de la población-, y deben poder rendir cuentas por ellos. En Argentina las redes de servicios de salud, desarrolladas en el subsector público, se implementaron como una estrategia para garantizar el acceso de la población a la atención integrando los distintos niveles de complejidad. Organizadas a partir de la Atención Primaria de la Salud, sostienen modelos productivos que concentran ciertos servicios y favorecen la dispersión de otros (Vilaça Mendes, 2013; Ariovich y Crojethovic 2015). 


\section{Referencias bibliográficas}

Ariovich, A. y Crojethovic, M. (2015). Las redes: un modelo organizativo para contrarrestar la fragmentación institucional del sistema de salud en la Argentina. Revista Gestión de las Personas y Tecnología, 8(24).

Ariovich, A. y Jiménez, C. (2014). Accesibilidad a la atención de la salud en el Municipio de Malvinas Argentinas. Los Polvorines: Universidad Nacional de General Sarmiento.

Blanco, J. (2009). Redes y territorios: articulaciones y tensiones. Actas del XII Encuentro de Geógrafos de América Latina-EGAL. Montevideo.

Brugué, Q.; Gomá, R. y Subirats, J. (2002). Conclusiones. En Subirats, J. (Coord.). Territorio y Gobierno. Nuevas respuestas locales a los retos de la globalización. Barcelona: Diputació de Barcelona.

Bonazzola, P. (2009). Ciudad de Buenos Aires: sistema de salud y territorio. Buenos Aires. Mimeo.

Chiara, M. (Comp.) (2012). Salud, politica y territorio en el Gran Buenos Aires. Los Polvorines: UNGS.

(2013). Politica de atención de la salud y relaciones intergubenamentales (RIG): continuidades e inflexiones en los patrones de interacción en el Gran Buenos Aires, Argentina (20II-20II). Tesis doctoral en Ciencias Sociales UBA. Buenos Aires.

Chiara, M.; Moro, J.; Ariovich, A.; Jiménez, C. y Di Virgilio, M. (201I). Lo local y lo regional: tensiones y desafíos para pensar la agenda de la política sanitaria en el conurbano bonaerense. En Chiara, M. y Moro, J. (Comps). Salud en las metrópolis. Desafíos en el conurbano bonaerense. Buenos Aires: UNGS- Prometeo Libros.

Dematteis, G. (2002). De las regiones-área a las regiones red. Formes emergentes de gobernabilidad regional. En Subirats, J. (Comp.). Redes, territorios y gobierno: nuevas respuestas locales a los retos de la globalización. Vol I. Barcelona: Xarxa de Municipis.

Dematteis, G. y Governa, F. (2005). Territorio y territorialidad en el desarrollo local. La contribución del modelo SLOT. Boletín de la Asociación de Geógrafos Españoles, (39).

Di Virgilio, M. (2003). Las estrategias desplegadas en relación a la salud y a la enfermedad. En Di Virgilio, M. Hábitat y Salud. Estrategias de las familias pobres. Buenos Aires: Lumiere.

Gutiérrez, A. (20II). Insumos para una gestión intersectorial de políticas públicas: movilidad y acceso. Territorios, 2(25), I5I-I72. 
(2012). ¿Qué es la movilidad? Elementos para (re) construir las definiciones básicas del campo del transporte. Bitácora Urbano Territorial, 2(2I). (20I3). Movilidad y territorio. Revisando el sesgo «materialista» en la gestión de políticas urbanas. Conferencia N-AERUS (Network-Association of European Researchers on Urbanisation in the South), Enschede, I2-I4 de septiembre.

Jirón Martínez, P. (2007). Implicancias de género en las experiencias de movilidad cotidiana urbana en Santiago de Chile. Revista venezolana de estudios de la mujer, I2(29). Caracas.

Lévy, J. (2009). Os novos espaços da mobilidade. GEOgraphia, 3(6). Universidade Federal Fluminense.

López, E. y Findling, L. (Coords.) (2012). Maternidades, paternidades, trabajo $y$ salud. ¿Transformaciones o retoques? Buenos Aires: Biblos.

Ministerio de Salud de la Provincia de Buenos Aires (2010). Guía de establecimientos 2010.

Ministerio de Salud de la Nación (20II). Utilización y gasto en servicios de salud en Argentina 20I0. Recuperado de: http:/www.msal.gob.ar/fesp/images/ stories/recursos-de-comunicacion/publicaciones/estudio_carga_enfermedad. pdf (acceso: I4 de abril de 20I7).

Ministerio de Transporte de la Nación (s/f). Encuesta de Movilidad Domiciliaria en el Área Metropolitana de Buenos Aires. Recuperado de: http://uecmovilidad.gob.ar/encuesta-de-movilidad-domiciliaria-2009-20Iomovilidad-en-el-area-metropolitana-de-buenos-aires/ (acceso: 8 de marzo de 20I7).

Organización Mundial de la Salud - Organización Panamericana de la Salud (2007). Renovación de la atención primaria de salud en las Américas: documento de posición de la Organización. Recuperado de: http://www2. paho.org/hq/dmdocuments/20Io/Renovacion_Atencion_Primaria_Salud_ Americas-OPS.pdf (acceso: 9 de octubre de 20I7).

Organización Panamericana de la Salud. - Ministerio de Salud de la Nación (20II). Regionalización de la atención perinatal. Documentos. Recuperado de: http://www.msal.gob.ar/images/stories/bes/graficos/ooooooor76cnt-no2regionalizacion.pdf (acceso: 30 de agosto de 2016).

Pozzio, M. (20II). Madres, mujeres y amantes: Usos y sentidos de género en la gestión cotidiana de las politicas de salud. Buenos Aires: Antropofagia.

Puebla, J. G. \& Palomares, J.C.G. (2006). Cambios en la movilidad en el área metropolitana de Madrid: el creciente uso del transporte privado. Anales de geografía de la Universidad Complutense, 25, 33I-35I. 
Santos, M. (1996). A natureza do espaço - Técnica e tempo, razão e emoção. São Paulo: Hucitec.

San Martín, M. y Luzuriaga, J.P. (2012). Estudio del perfil de complejidad de los hospitales provinciales de la provincia de Buenos Aires, Argentina. Buenos Aires: Ministerio de Salud de la Provincia de Buenos Aires.

Silveira, R.D. (2003). Redes e território: uma breve contribuição geográfica ao debate sobre a relação sociedade e tecnologia. Biblio 3 W, Revista Bibliográfica de Geografia y Ciencias Sociales, Universidad de Barcelona, VIII(45I).

Vilaça Mendes, E. (2013). Las redes de atención de salud. Brasil: Organización Panamericana de la Salud. 